\title{
Fish harvesting strategies with allee effect
}

\author{
Ashutosh Maurya ${ }^{1, *}$ and Anupam Priyadarshi ${ }^{1}$ \\ ${ }^{1}$ Department of Mathematics, Institute of Science, Banaras Hindu University, Varanasi, India
}

\begin{abstract}
Fish harvesting is important for nutritious food availability in human diet. In this manuscript, a simple fish harvesting model is developed using logistic growth along with allee effect. We study the basic mathematical properties of the model as equilibrium analysis and stability. For better understanding of the model performance, we perform the simulation of the system with different constant harvesting rate and periodic harvesting rate. The results shows that periodic harvesting is economically beneficial than the constant harvesting in fish stock.
\end{abstract}

Keywords: Fish stock, Sine periodic harvesting, Economic beneficial, Allee effect, Allee threshold.

\section{Introduction}

Fish is broadly acceptable human diet. It is not only diet but also the main source of protein, fat and vitamin [1]. Daily use of meat increases low-density lipoprotein cholesterol which causes cardiovascular arrest in the human body. However, consumption of fish increases high-density lipoprotein cholesterol and omega-3 fatty acids and lowers the triglycerides level which improves human healthy life. Awareness towards fish consumption as a nutritious diet has increased the demand of fish for food consumption. Supply of fish cannot rely on the ocean fish activities thus alternatives can be found by communicatively the agriculture. Fisheries are worldwide and they have different harvesting strategies like partial harvesting and total harvesting [2]. Partial harvesting is the harvesting of a specific age or size group fish at a particular time while total harvesting is complete cropping at end of tenure. Along with these harvesting techniques, some other approaches are also used like periodic harvesting and another functional type in theoretical mathematical modelling [2]. These studies are required for the improvement in fish stocks and also increment in income of farmers due to short term market volatility.

Mathematical models have been used widely to estimate the population dynamics of animals for so many years as well as the human population dynamics [3]. In recent years, the use of mathematical models has been included in the agriculture sector especially in cattle forming to ensure confirmation and optimum supply. The basic functional response logistic growth widely applied with an ordinary differential equation for manipulating the species population. There is various type of traits that are present in the real scenario but our attentions are limited. The smaller population size is eliminated in surrounding in very less time. This situation in mathematical terms known as ALLEE EFFECT and given by

\footnotetext{
*e-mail: ashunano@gmail.com
} 
W. C. allee in 1931 by experimental experiences. Allee effect is one of the basic traits which is applicable in low population [4]. So, it is required to apply Allee effect term in fish harvesting models.

The most important thing for successful management of harvested population is that the harvested strategies are sustainable not leading to instabilities or extinctions and produces a great result for the year with the variation between the year [2]. So, it can be supplied throughout the year smoothly. Laham et al.[6] used data of tilapia fish from Malaysia fisheries department and make a mathematical model with the logistic growth rate. In their study, they considered constant harvesting rate and periodic harvesting rate. Prey- predator traits are responsible for qualitative behaviour of the Model. Introducing a new traits may alter the system dynamics qualitatively. In our work, we extend their studies by introducing allee effect trait in growth rate with constant harvesting rate and periodic harvesting rate and deduce that which one is better economically. As per our knowledge we are the first who applied allee effect in the real data in Ordinary Differential Equation and comprise the result with sine periodic harvesting. As an application point of view, the proposed model can be helpful to the fish farming sector and fisheries department to estimate the fish population.

The objective of this study is to maximize the fish yield through the implemented harvesting strategies. Further an objective is to know the optimum quantity of harvesting that can ensure the continuous market supply without elimination. Finally, we compare the results obtained between the two strategies. The strategies would be guaranteed the supplies are nonstop.

\section{Model and Mathematical Analysis}

The initial data for the present study has been carried out by the paper Lakam et. al.[6]. The Fisheries of Malaysia (2008) surveyed $15.61 \mathrm{Ha}$. with carrying capacity of high population 780500 fish and 5 tilapia fish can sustain for every $1 \mathrm{~m}^{2}$ surface area. They also confirm that fish matures in 6 months and $80 \%$ fish will be mature [7]. However, at low populations, the fisheries can be extinct. To circumvent this, we have applied the allee effect term with logistic growth and described in the following section.

\subsection{Modified logistic growth along with the allee effect of fish with constant harvesting (Model 1)}

We consider a single species mathematical model as an ordinary differential equation (see equation1) of fish harvesting using logistic growth along with allee effect in this study. In the model, $p$ denotes the fish density, $r$ is the growth rate, $k$ is carrying capacity of ponds. Logistic growth of fish population is given by $\frac{d p}{d t}=r p\left(1-\frac{p}{k}\right)$. Fish population is not survived below a threshold population which is called allee threshold $A$. This allee effect is applied in logistic growth fish is given by $\frac{d p}{d t}=r p\left(1-\frac{p}{k}\right)\left(\frac{p}{k}-\frac{A}{k}\right)$. Let constant harvesting $H$ is occurring in the pond. The mathematical model for the aforementioned system is described as follows (Model 1):

$$
\frac{d p}{d t}=\operatorname{rp}\left(1-\frac{p}{k}\right)\left(\frac{p}{k}-\frac{A}{k}\right)-H
$$

The equilibrium of Model1 is determine by

$$
A_{1} p^{3}+B p^{2}-C p-H=0
$$


where

$$
A_{1}=-\frac{A r}{k^{2}}, B=\frac{r}{k}+\frac{A r}{k^{2}}, C=\frac{A r}{k}
$$

The equilibrium points are given by

$$
\begin{gathered}
E_{1}=q_{1}-\frac{B}{3 A}+\frac{q_{2}}{q_{1}} \\
E_{2}=-\frac{q_{1}}{2}-\frac{B}{3 A}-\frac{q_{2}}{2 q_{1}}-i \sqrt[3]{\left(q_{1}-\frac{q_{2}}{q_{1}}\right)} \\
E_{3}=-\frac{q_{1}}{2}-\frac{B}{3 A}-\frac{q_{2}}{2 q_{1}}+i \sqrt[3]{\left(q_{1}-\frac{q_{2}}{q_{1}}\right)}
\end{gathered}
$$

Where

$$
\begin{gathered}
q_{1}=\sqrt[3]{\left(\frac{H}{2 A}-\frac{B^{3}}{27 A^{3}}+\sqrt{\left(\frac{B^{3}}{27 A^{3}}-\frac{H}{2 A}+\frac{B C}{6 A^{2}}\right)^{2}-q_{2}^{3}}-\frac{B C}{6 A^{2}}\right)} \\
q_{2}=\frac{C}{3 A}+\frac{B^{2}}{(3 A)^{2}}
\end{gathered}
$$

Theorem 2.1 Assume $J=3 A_{1} p^{2}-2 B p-C$ (i) If $J<0$ holds then $E_{1}, E_{2}$ and $E_{3}$ equilibrium points of model 1 always exist and asymptotic stable. (ii) If $J>0$ holds then $E_{1}, E_{2}$ and $E_{3}$ equilibrium points of model 1 always exist and unstable. (iii) If $J=0$ holds then $E_{1}, E_{2}$ and $E_{3}$ equilibrium points of model 1 always saddle.

Due to weather and other reason, Market has not a uniform demand of fish. And fish has also breeding seasons so we avoid in this time to harvest. So we have desire other harvesting strategies which are more beneficial to the farmer in short term. So we applied periodic harvesting (in sine terms) in equation 1.

\subsection{Modified logistic growth along with the allee effect of fish with periodic harvesting (Model2)}

We reforming the previous model with sine harvesting according to the need of the market. The Model 2 is given by

$$
\frac{d p}{d t}=r p\left(1-\frac{p}{k}\right)\left(\frac{p}{k}-\frac{A}{k}\right)-\frac{p|\sin (\pi t)|}{4}
$$

Description of parameter are aforementioned. The equilibrium point of $\operatorname{Model}(2)$ is given by the equation $A_{1} p^{3}+B p^{2}-C p=0$ Where $A_{1}=-\frac{r}{k^{2}}, B=\frac{r}{k}+\frac{r A_{1}}{k^{2}}, C=\frac{r A_{1}}{k}+\frac{|\sin (\pi t)|}{4}$ After calculations, we got the equilibrium points as $E_{4}=0, E_{4,5}=\frac{-B \pm \sqrt{B^{2}+4 A_{1} C}}{2 A_{1}}$

Theorem 2.2 Assume $J=3 A_{1} p^{2}-2 B p-C$ (i) If $J<0$ holds then $E_{4}, E_{5}$ and $E_{6}$ equilibrium points of model(2) always exist and asymptotic stable. (ii) If $J>0$ holds then $E_{4}, E_{5}$ and $E_{6}$ equilibrium points of model(2) always exist and unstable. (iii) If $J=0$ holds then $E_{4}, E_{5}$ and $E_{6}$ equilibrium points of model(2) always saddle. 


\section{Simulations}

For detailed analysis, we use XPPAUT, MATHEMATICA AND MATLAB Software for simulation. We have taken parameter values as initial population $p=780500$, growth rate $r=0.8$, carrying capacity $k=780500$, allee threshold $A=0.01$. We observe that the dynamical system is stable for constant population $H=90000$, which implies that the fish species are survived for long years (See Figure 1). On the other hand, if we take harvesting rate $H=95000$, the fish species are not survived for the 60 unit time, which means they might be eliminated through this harvesting rate.

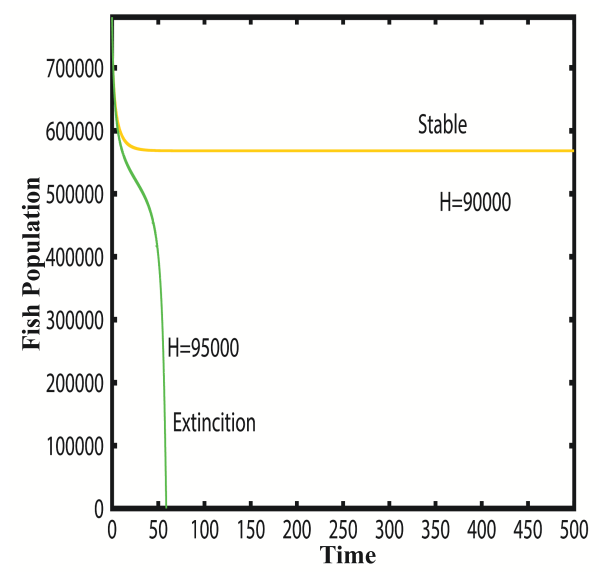

Figure 1. Stability and extinction line for Constant harvesting rates.

In a natural scenario, the constant harvesting is not possible due to market needs and it is not sustainable so we proceed to periodic harvesting.

$$
\frac{d p}{d t}=r p\left(1-\frac{p}{k}\right)\left(\frac{p}{k}-\frac{A}{k}\right)-\frac{p \sin (\pi t)}{q}
$$

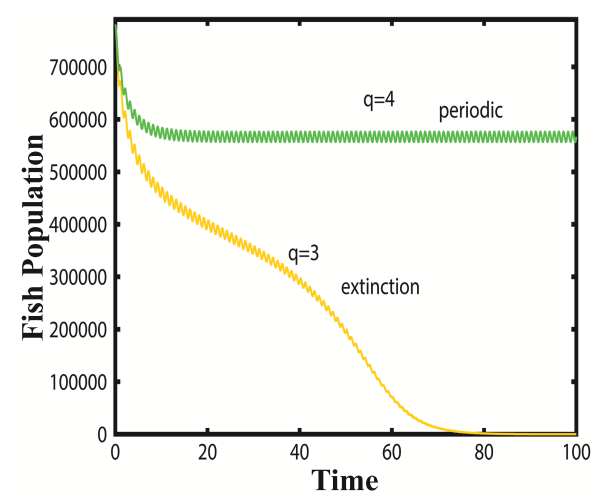

Figure 2. Periodic population and extinction with the different value of $q$.

Since there is breeding time for fish and we want to avoid harvesting in breeding season so we take $\sin (\pi t)$ type function. We notice that when $q=4$, it gives suitable results for 
fish population survive periodically and at $q=3$ fish species are extinct. In Figure 2, we have drawn the graph at the equilibrium condition of Model 2 to see the effect of harvesting. We have seen that at $q=4$ it attained Maximum fish harvesting near 25\% of the total fish population or in this initial population it would be near about 194260 (see in Figure 3) Further, we integrate Ordinary Differential Equation and see the effect of this periodic harvesting rate for population dynamics. We have found the system is sustainable with periodic population dynamics (see figure 4).

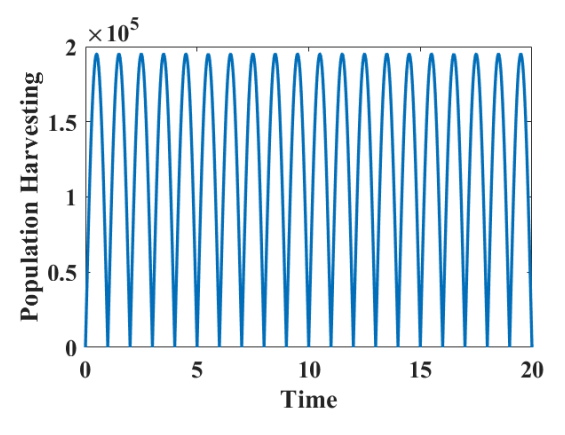

Figure 3. harvesting effect at $\frac{d p}{d t}=0$ at $q=4$ in model 2

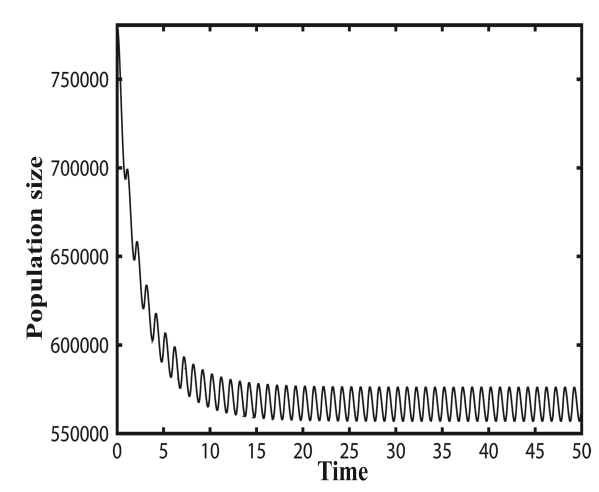

Figure 4. Fish population dynamics for $q=4$ in model 2 .

\section{Important Results and discussion}

We use different traits like allee effect and constant harvesting in the logistic growth model which is not previously used for this type of fish data in the literature. The constant harvesting model with allee effect can show only two types of qualitative behavior like stable fish population and extinction of the entire fish population. While the second periodic model with sine culling shows periodic population behavior and extinctions. In this scenario, we can manage the fish population and culling according to the market so that the farmers can be fruitful in making money and profit through the market. There is always fish stock is available in the pond as it gives periodic solutions. It is a low cost occurring since the pond is not drained completely at any time.

\section{Conclusion:}

In the end, we conclude that the optimize solution found with the constant and periodic solution. These harvesting strategies using periodic harvesting can be used to improve production shorten investment, return time and reduce the risk of changes in selling price and cost price, particularly when comparatively short return period is used. However, using constant harvesting the pond can be empty through higher harvesting rate. This can improve the returns of the farmer before harvesting. This study can help fisheries in freshwater pond growing as agriculture activity under conclusion.

\section{Acknowledgement}

The first author acknowledged to CSIR, New Delhi for providing Junior Research fellowship wide fileno. $-9 / 013(0822) / 2018-E M R-1$. 


\section{References}

[1] M. C. Nesheim , M. Oria ,P. T. Yih , Institute of Medicine; National Research Council; editors,. Washington (DC): National Academies Press (US); 2015 Jun 17. Available from: https://www.ncbi.nlm.nih.gov/books/NBK305181/ doi: 10.17226/18846

[2] L.V. Idels, and M. Wang, International Journal Modelling, Identification and Control 3, 83-87,2008.

[3] J.D. Murray, Mathematical Biology 1: An Introduction, Springer Verlag,USA, 1993.

[4] H. U. Wittmer, F. Courchamp, L. Berec, and J. Gascoigne, Allee effects in ecology and conservation, Oxford University Press, New York, 272 pp. ISBN 978-0-19-956755-3, (2008)

[5] S. Aanes, S. Engen ,B-E. Saethe, T. Willerbrand, and V. Marcstram, Ecological Applications 12, 281-290 (2002).

[6] M. F. Laham, I.S. Krishnarajah and J. M. Sharief Sains Malaysiana 41(2), 171177 (2012).

[7] P. Thomas, and M. Michael, Tilapia life history and Biology., Southern Regional Aquaculture Center 283, 1999. 